\title{
FUZZY-LOGIC-BASED TCP CONGESTION CONTROL SYSTEM
}

\author{
A. M. Al-Naamany and H. Bourdoucen \\ Sultan Qaboos University, College of Engineering \\ Information Engineering Department, PO Box 33, Al Khod, 123 \\ Muscat, OMAN Emails: naamany@squ.edu.om, hadj@squ.edu.om
}

\begin{abstract}
The Internet and most current intranet networks are experiencing a huge increase in the volume of traffic. This affects directly the network congestion by saturating the buffers at the routers and contributes to generating lots of data losses as well as reception and transmission delays. The existing TCP end-to-end congestion control uses Additive Increase Multiplicative Decrease (AIMD) approach, a time out and slow start behavior, which lead to data throughput with abrupt changes. Therefore, developing new congestion control strategies based on non-analytical approaches will certainly help to overcome the current difficulties of the internet in particular which are due to network structural complexity, diversity of services supported, and to variety of parameters involved. This work presents a fuzzy logic-based approach for controlling the network congestion. Its main objective is to optimize the available bandwidth and keep smooth the data throughput transfer profile.
\end{abstract}

Key words: Fuzzy logic, Congestion Control, TCP, bandwidth optimization

\section{INTRODUCTION}

The Internet is currently experiencing a huge increase in the volume of data transfer, which in most of the time exceeds the available network resources [1],[2],[3],[4],[5],[6]. This is also the trend for wide area networks (WANs). This is mainly due to different applications that run concurrently and require high rate of data transfer [3],[4],[5],[6],[7]. The TCP protocol, which is the most popular protocol used for data exchange through the Internet, controls the congestion using the measured packet loss rate. It uses

The original version of this chapter was revised: The copyright line was incorrect. This has been corrected. The Erratum to this chapter is available at DOI: 10.1007/978-0-387-35703-4_21

D. Gaïti et al. (eds.), Network Control and Engineering for QoS, Security and Mobility II

(C) IFIP International Federation for Information Processing 2003 
what is known as the Additive Increase Multiplicative Decrease (AIMD) approach. It consists of reducing the transmission rate by a factor of two whenever a packet loss is signaled and increases the transfer rate when no loss is declared [9],[10],[11],[12]. This congestion control approach does not seem amenable to provide promising solution for scalable multicast congestion control. In addition to this and based on many published papers, this control approach has a data transfer profile which is characterized by many abrupt changes. These changes in the sending rate have been a key impediment to the deployment of this approach to end-to-end congestion control by emerging new Internet applications such as streaming multimedia. An alternative approach known as equation-based congestion control has been proposed to provide smooth congestion of the control profile [3]. On the other hand, a significant research on equation-based as well as on other congestion control approaches for best-effort streaming multimedia has been published in the past few years [13],[14],[15],[16],[17],[18]. The equation-based congestion control algorithm, first proposed by J. Mahdavi and S. Floyd in 1997, gives explicitly the maximum acceptable sending rate as a function of the measured recent loss event rate [3]. Based on this value, the sender adapts its sending rate, which is guided by a control equation relating the transmission rate to the loss event rate, the size of data packet, and the round trip time. This technique was judged to be one of the leading candidates for congestion control and a viable technique, which provides relatively smooth congestion control, compared to TCP.

This work aims to achieve smoothing of the sending data rate profile while optimizing the use of the available network bandwidth. To achieve this objective, a fuzzy logic compensator is utilized to identify the transfer rate steps depending on the packet drop rate and the round trip time. The adequate bandwidth and throughput profile are therefore, approached slowly, and tracked as the congestion parameters change. This method is proposed as means to reduce unnecessary transmission, which cannot be received due to dynamic network constraints. Thus, the present paper tends to optimize both, the data throughput ripple as well as the network available bandwidth. This is performed using a non-analytical congestion avoidance approach based on fuzzy logic theory.

\section{DESIGN AND SET-UP OF FUZZY LOGIC CONTROLLER}

The fuzzy logic controller that has been implemented for congestion control is shown in Figure 1. It is built around a fuzzy logic compensator used 
to identify the compensation value $\boldsymbol{f}_{T}$, which is used to adjust the sending rate $\mathbf{T}$ to its optimal value depending on the network load and performance. This optimal value is approached in factors that are identified by the Fuzzy compensator using round trip time $\mathbf{R}$ and steady state packet loss event rate p. To smooth the rate of transmitted data, a damping mechanism is utilized. It has a variable scaling factor $\mathbf{k}$ used to damp any abrupt changes of the transmission rate $\mathbf{T}$.

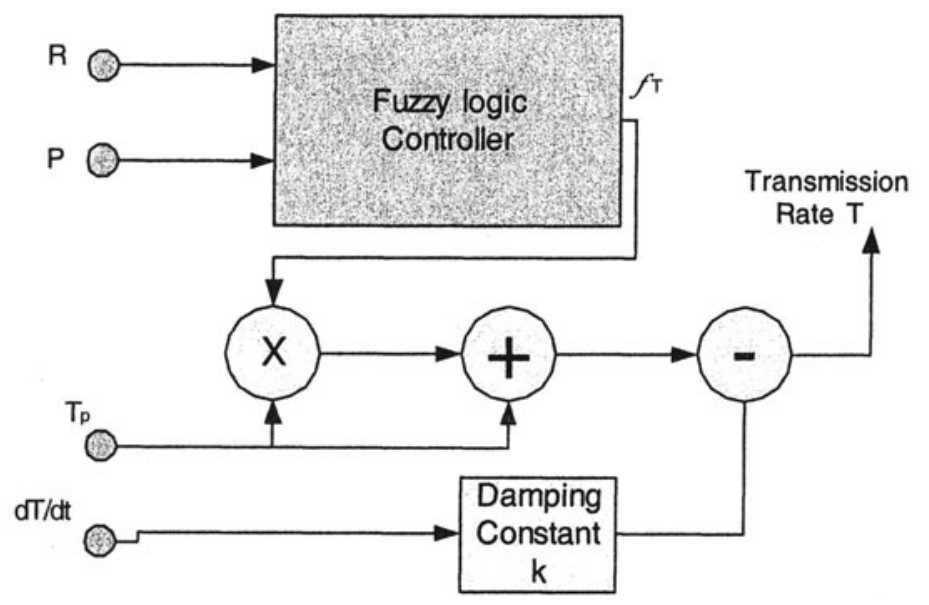

Figure 1 Block Diagram of the fuzzy logic model for congestion control

In this case the sending rate is adjusted as follows:

$$
T=T_{p}\left(1+f_{T}\right)-k \frac{\left(T_{p} f_{T}\right)}{\Delta t}
$$

Where $\boldsymbol{f}_{\boldsymbol{T}}$ is the output of the fuzzy logic compensator, $\boldsymbol{T}_{\boldsymbol{p}}$ is the previous sending rate while $\Delta t$ is the period since the last calculated sending rate and $\boldsymbol{k}$ is the damping factor.

The fuzzy compensator is shown in Figure 2. The fuzzifier transforms the crisp data of the round trip time $\mathbf{R}$ and the steady state loss event rate $\mathbf{p}$ into fuzzy sets by evaluating the membership functions. The fuzzy rule base contains a set of fuzzy rules in linguistic form, which are used to manipulate the fuzzy algorithms. Inference engine evaluates the rules and based on the preconditions recommends a set of compensation to the sending rate $\mathrm{T}$. The 
overall fuzzy output is the union of the outputs resulting from each rule. The defuzzification produces a non-fuzzy compensation action that best represents the adequate sending rate.

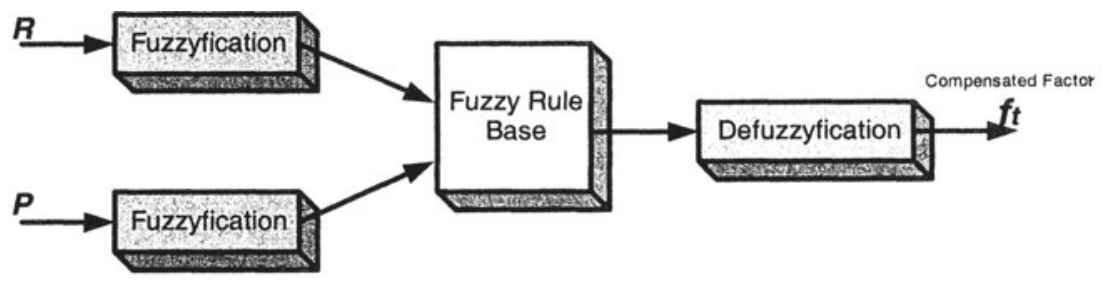

Figure 2 Fuzzy logic Controller

The fuzzifier performs a mapping from a crisp point $\bar{X}=\left(\mathrm{x}_{1}, \ldots . . \mathrm{x}_{\mathrm{n}}\right)^{\mathrm{T}} \in \mathrm{U}$ ( universe of discourse) into a fuzzy set $A^{\prime}$ in $U$. We will use the singleton fuzzifier (the most commonly used), which is by definition, $A^{\prime}$ is a fuzzy singleton with support $\overline{\mathrm{x}}$, that is, $\mu_{\mathrm{A}^{\prime}}\left(\overline{\mathrm{X}}^{\prime}\right)=1$ for $\overline{\mathrm{X}}^{\prime}=\overline{\mathrm{X}}$ and $\mu_{\mathrm{A}^{\prime}}\left(\overline{\mathrm{X}}^{\prime}\right)=0$ for all other $\overline{\mathrm{X}}, \in U$ with $\overline{\mathrm{X}}, \neq \overline{\mathrm{X}}$. The membership function for each term in the partitioned spaced is expressed mathematically as

$$
\mu_{\mathrm{A}}(\mathrm{x})=\left\{\begin{array}{cc}
0 & \mathrm{x} \leq \mathrm{a} \\
\frac{\mathrm{x}-\mathrm{a}}{\mathrm{b}-\mathrm{a}} & \mathrm{a} \leq \mathrm{x} \leq \mathrm{b} \\
\frac{\mathrm{c}-\mathrm{x}}{\mathrm{c}-\mathrm{b}} & \mathrm{b} \leq \mathrm{x} \leq \mathrm{c} \\
0 & c \leq x
\end{array}\right.
$$

Where $\mathrm{a}, \mathrm{b}$ and $\mathrm{c}$ are the three corners of the triangular with $\mathrm{a}<\mathrm{b}<\mathrm{c}[19]$.

The two variables to be considered are round trip time $\mathbf{R}$ and the steady state loss event rate $\mathbf{p}$. 

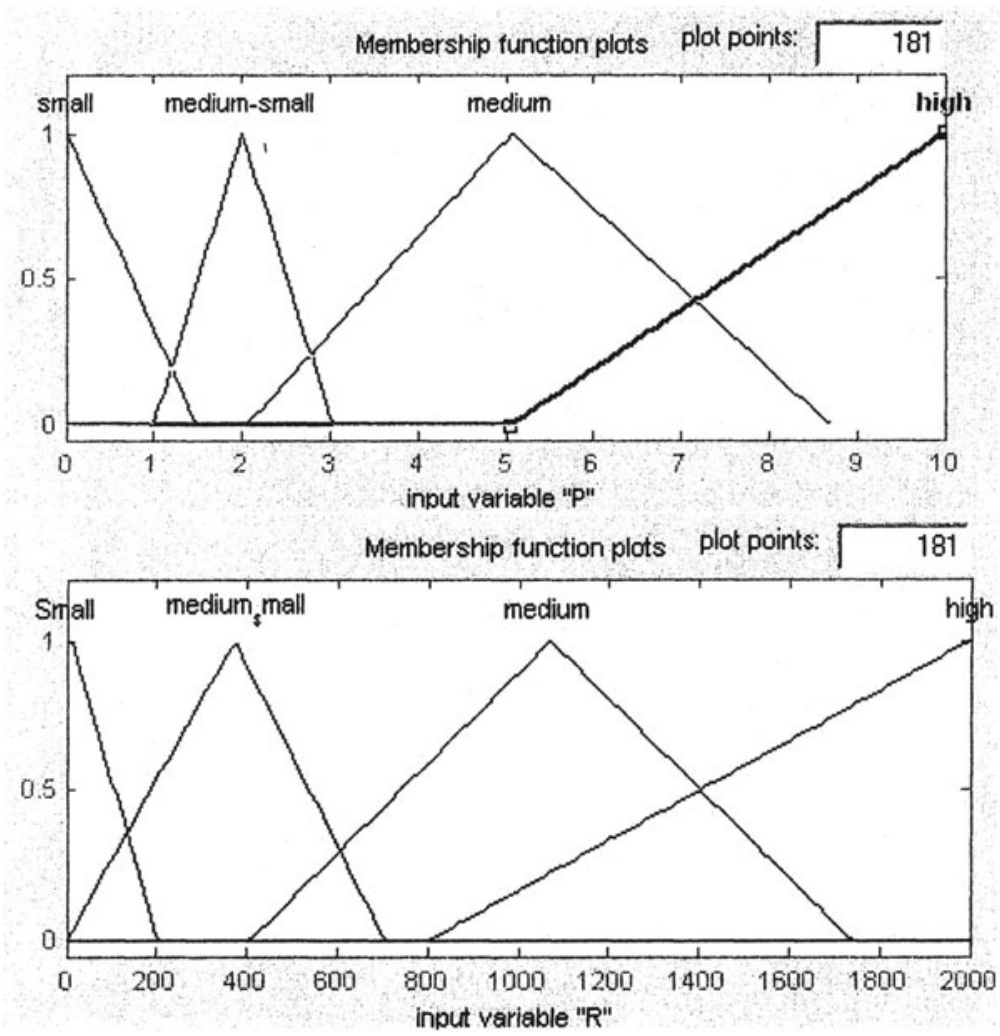

Figure 3 Fuzzy logic membership functions used to simulate the model

Each variable is separated into four input membership functions, small, medium-small, medium and high which represents their numeric values as input to the fuzzy controller.

A representation of the round trip time $\mathbf{R}$ input membership function domain and steady state loss event rate $\mathbf{p}$ is given in Figure 3 . The number of memberships is same however their positioning is to allow emphasis on the effects of packet loss in comparison to round trip time. The effect of packet loss rate $\mathbf{p}$ and round trip time $\mathbf{R}$ on fuzzy logic transmission rate change $\mathrm{f}_{\mathrm{T}}$ is shown in Figure 4. 


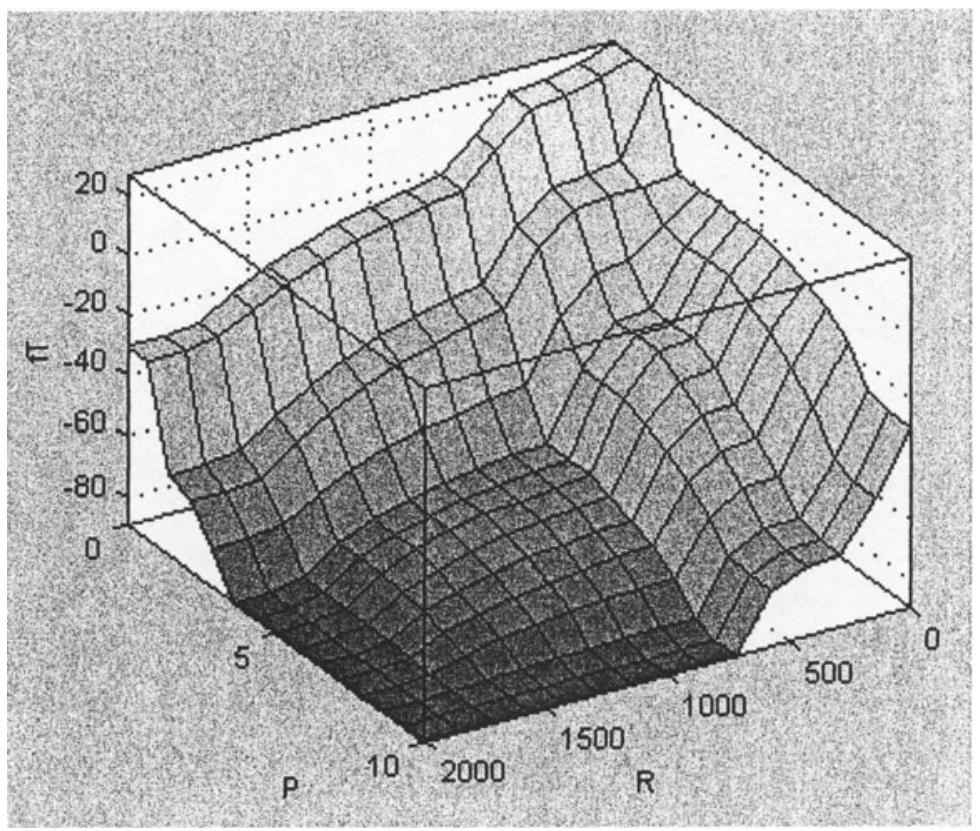

Figure 4 Effect of packet loss rate $\mathrm{p}$ and round trip time $\mathrm{R}$ on fuzzy logic transmission rate change $\mathrm{f}_{\mathrm{T}}$

The control rule base is set up using IF-THEN rules based on the fuzzy model. The IF-THEN rules describe what action is to be taken if a certain set of conditions is met. It incorporates information about every possible condition that the system can encounter. Note that when a fuzzy rule base recognizes information that is partially true it can partially invoke more than one rule at a time. If two or more rules are to be invoked that have the same value, than the rule that is invoked to the greatest degree is chosen (the maximum), and the rest are discarded. This is the union fuzzy operation. The fuzzy logic rule base is represented as a two-dimensional table look up in Figure 5. 


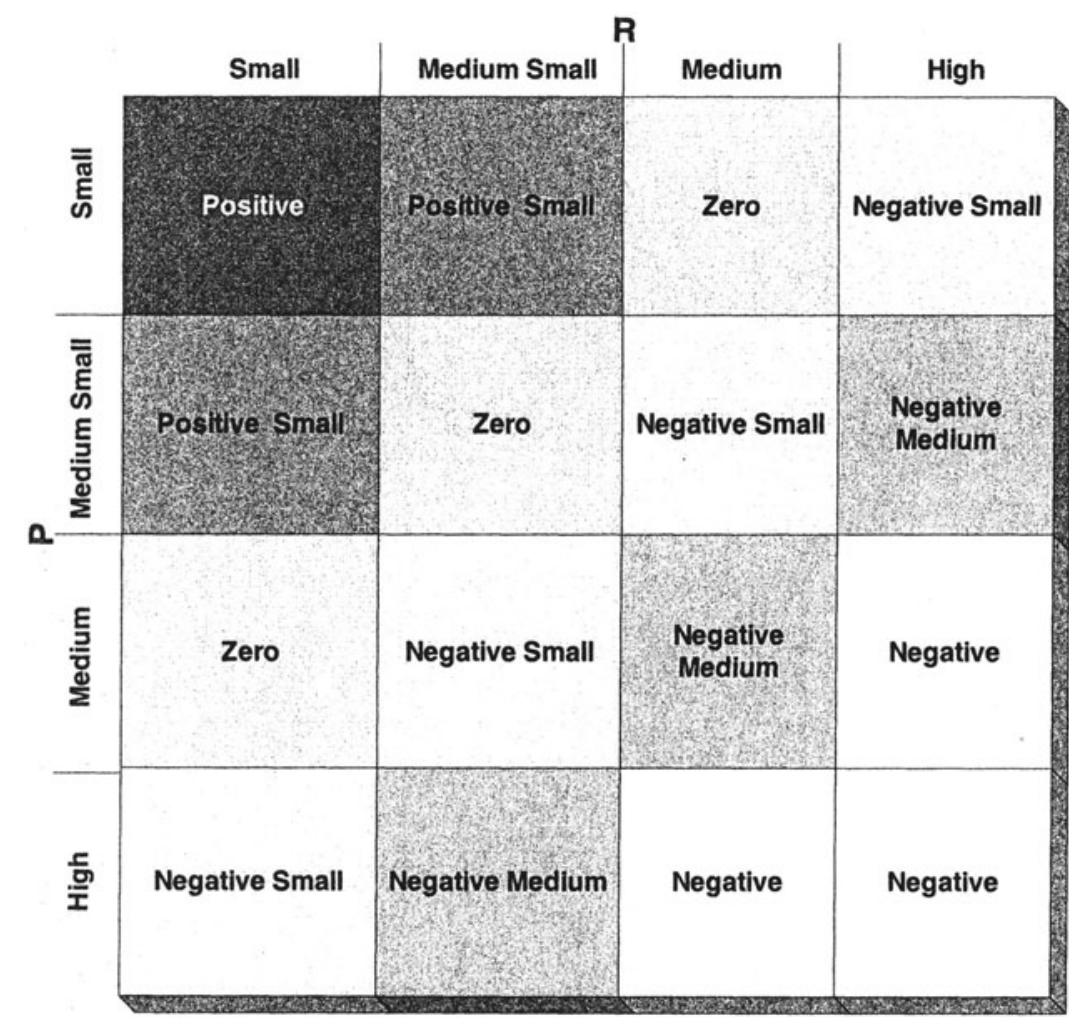

Figure 5 Fuzzy logic rule

In this method the fuzzy logic controller produce an output as number ranging from negative to a positive number representing the multiplicative factor of the sending rate $T$.

In evaluating the rules, we used the minimum operator (fuzzy AND operator), i.e.

$\mu^{\mathrm{i}} \mathrm{O}^{\mathrm{i}}=\mu_{\mathrm{r}_{1}^{\mathrm{i}}}\left(x_{1}\right) \wedge \mu_{\mathrm{r}_{2}^{\mathrm{i}}}\left(x_{2}\right)$, where $O^{\mathrm{i}} \quad$ denotes the output region of rule $\mathrm{i}$, and $I_{j}^{i}$ denotes the input region of rule $i$ for the $j$ components, $\mathrm{x}_{1}$ and $\mathbf{x}_{2}$ are the two inputs, $\mathbf{P}$ and $\mathbf{R}$ in our case [19],[20],[21].

For the defuzzification, an average defuzzification formula is used to determine the outputs [19]. Where $j$ denotes the $\mathrm{j}^{\text {th }}$ component of the output vector $\left(\mathrm{O}_{\mathrm{j}}^{\mathrm{i}}\right.$ is the region of Rule $i$ for the $\mathrm{j}^{\text {th }}$ output component, 


$$
y_{i}=\frac{\sum_{i=1}^{M} \mu_{o_{j}^{i}}^{i} \overline{\mathrm{y}}_{\mathrm{j}}^{\mathrm{i}}}{\sum_{i=1}^{M} \mu^{i} o_{j}^{i}}
$$

$\bar{y}_{j}^{i}$ denotes the center value of region $O_{j}^{\mathrm{i}}, \mathrm{M}$ is the number of fuzzy rules in the combined fuzzy rule base and hence the output of the defuzification stage. This output is crisp value denoting the response of the fuzzy logic controller. In this case, the transfer rate $T$ is the output of this stage of controller, which is used for the next data transfer rate.

\section{ANALYSIS AND RESULTS}

Figure 6 shows the expected results of the fuzzy logic controller with and without damping function. The controller is expected to react rapidly to any change in the round trip time $\mathbf{R}$ and the steady state loss event rate $\mathbf{p}$, which, once the actual bandwidth is reached, can cause oscillations. These oscillations are mainly due to the large sampling period. However, the implementation of a damping function on the output of the fuzzy logic compensator reduces the effect of large changes due to minor variations in the input. This results in a slowed down reaction of the overall controller, hence, a reduction in amplitudes of oscillations once the actual bandwidth is reached. 


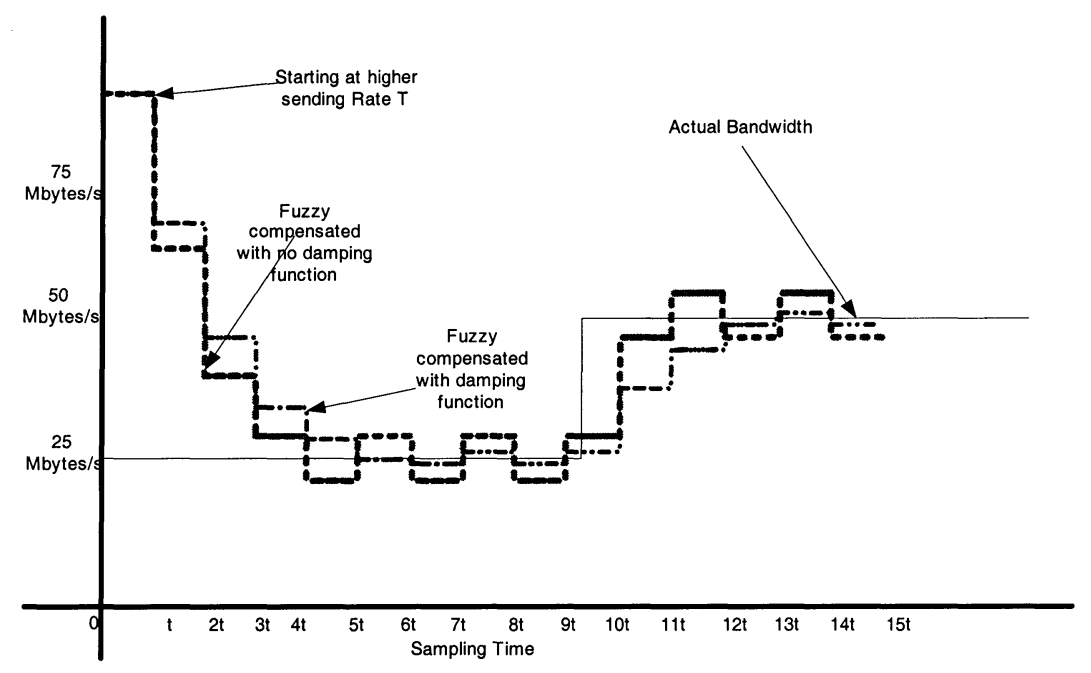

Figure 6 Effect of Fuzzy Compensator with and without damping function

To reduce the congestion effect further, we are suggesting adding increment/decrement steps to approach the next sending rate. In this case, once the next sending rate $\mathbf{T}$ is computed, the system should not jump to this new sending rate by one single step. Instead, $\mathbf{n}$ number of step should be used to approach this new sending rate within the sampling period. By undertaking this, the system will smoothly seek the optimal bandwidth without excessively inducing congestion by sending large quantity of packets, which cannot be handled by the system or accepted by the receiver. Figure 7 depicts the expected profile that results when using $\mathbf{n}$ step adjustments approach.

\section{CONCLUSION}

A fuzzy logic TCP congestion controller has been proposed in this paper. It aims to smooth the data throughput profile and to optimize the network transfer bandwidth. This has been achieved using a fuzzy logic compensator, which identifies the transfer rate steps depending on the packet drop rate and the round trip time of the network at a given point of time. In addition, due to dynamic network constraints, the proposed method allows proximity tracking of both the bandwidth and data throughput profile. This has been ensured by embedding within the controller a damping function to avoid abrupt changes of the transmission rate. Thus, this approach allows reducing unnecessary over-transmitted packets that will be lost in the network and at the same time reduces the ripple in the throughput profile. Because of its non-analytical nature, the proposed approach appears as a 
good candidate to complement the available congestion controllers for unicast as well as for multicast systems.

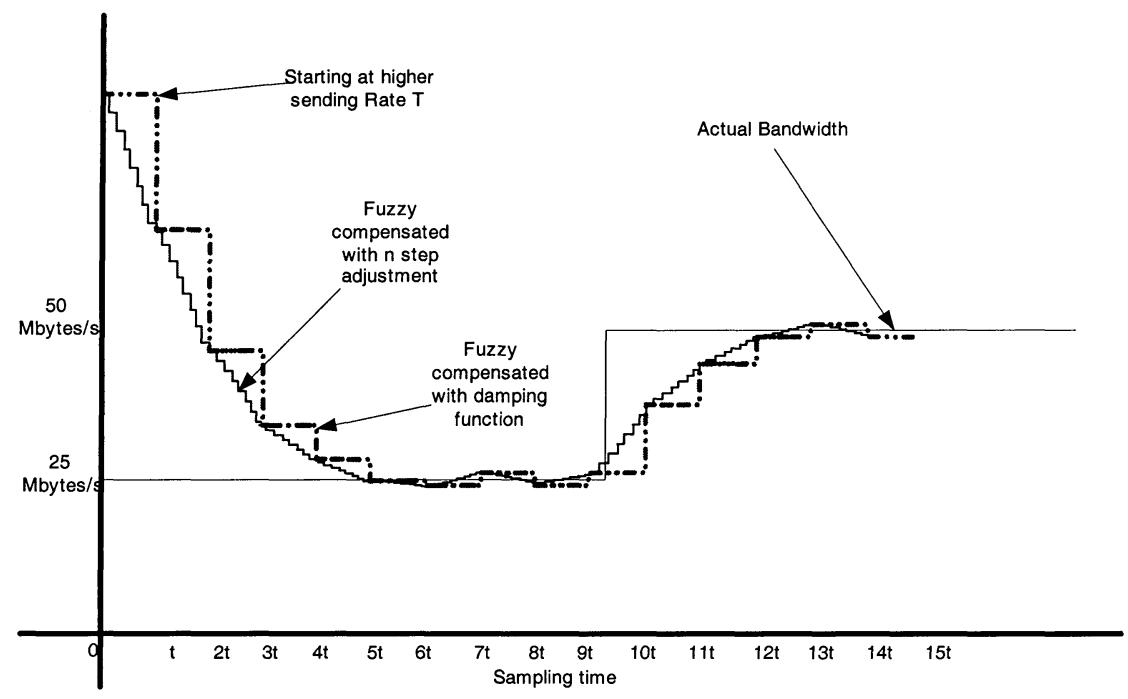

Figure 7 Effect of Fuzzy Compensator with n step adjustments

\section{REFERENCES:}

[1] S. Floyd, M Handely, J Padhye and J Widmer; " Equation-based congestion control for unicast applications: the extended version", International Computer Science Institute tech report TR-00-03, March 2000.

[2] M. Allman et al., "Ongoing TCP research related to satellites," RFC 2760, Feb. 2000.

[3] J. Padhye and S. Floyd, TBIT Web site: http://www.aciri.org/tbit// and the references therein.

[4] S. Floyd, "A report on recent developments in TCP congestion control", IEEE Communications Magazine, April 2001.

[5] H. Balakrishnan et al., "TCP behavior of a busy web server: analysis and improvements," IEEE INFOCOM, Mar. 1998.

[6] M. Allman, H. Balakrishnan, and S. Floyd, "Enhancing TCP's loss recovery using limited transmit," RFC 3042, Jan. 2001. 
[7] S. Floyd et al., "An extension to the selective acknowledgement (SACK) option for TCP," RFC 2883, July 2000.

[8] J. Padhye, V. Firoiu, D. Towsley and J. Kurose; “ Modeling TCP throughput: a simple model and its empirical validation", UMASS CMPSCI Tech Report TR98008, Feb. 1998.

[9] M. Mathis et al.,"TCP selective acknowledgment options," RFC 2018, Apr. 1996.

[10] J. Stone and C. Partridge, "When the CRC and TCP checksum disagree," SIGCOMM Symp. Commun. Architectures and Protocols, Sept. 2000.

[11] S. Floyd and V. Jacobson, "On traffic phase effects in packet-switched gateways," Internetworking: Research and Experience, vol. 3, no. 3, Sept. 1992, pp. 115-56.

[12] S. Floyd and V. Jacobson, "Random early detection gateways for congestion avoidance,” IEEE/ACM Trans. Net., vol. 1, no. 4, Aug. 1993, pp. 397-413.

[13] S. Jacobs and A. Eleftheriadis. "Providing video services over networks without quality of service guarantees". In World Wide Web Consortium Workshop on RealTime Multimedia and the Web, 1996.

[14] R. Rejaie, M. Handley, and D. Estrin. "An end-to-end rate-based congestion control mechanism for real time streams in the internet". INFOCOMM 99 Proceedings, 1999.

[15] J. Padhye, J. Kurose, D. Towsley, and R. Koodli. "A model based tcp-friendly rate control protocol”. NOSSDAV'99 Proceedings, 1999.

[16] T. Turletti, S. Parisis, and J. Bolot. "Experiments with a layered transmission scheme over the internet". Technical report RR-3296, INRIA, France.

[17] L. Vicisano, L. Rizzo, and J. Crowcroft. "TCP-like congestion control for layered multicast data transfer”, INFOCOMM'98 In Proceedings, 1998.

[18] D. Sisalem and H. Schulzrinne. "The loss-delay based adjustment algorithm: a TCPfriendly adaption scheme”, NOSSDAV'98 Proceedings, 1998.

[19] J. Jang, "Neuro-Fuzzy and soft computing" Prentice Hall, New Jersey, USA 1997.

[20] C. J. Harris, C. G. Moore, and M. Brown, "Intelligent control, aspects of fuzzy logic and neural networks", 1993, World Scientific.

[21] L. Wang, "Adaptive fuzzy systems and control, design and stability analysis"; 1994, Prentice Hall. 